\title{
Prevalencia y susceptibilidad de Cryptococcus neoformans aislados del suelo y excretas de palomas en la ciudad de Maceió - AL, Brasil
}

\author{
Prevalência e suscetibilidade de Cryptococcus neoformans isolados do solo e de \\ excretas de pombos na cidade de Maceió - AL, Brasil
}

\author{
Rodrigo José Nunes Calumby ${ }^{1 *}$, Davi Porfirio da Silva², Rossana Teotônio de Farias Moreira ${ }^{3}$, Juliane \\ Cabral Silva ${ }^{4}$, Luciano Aparecido Meireles Grillo ${ }^{5}$, Maria Anilda dos Santos Araújo ${ }^{6}$
}

\begin{abstract}
${ }^{1}$ Mestrando do Programa de Pós-graduação em Ciências Farmacêuticas, Universidade Federal de Alagoas, UFAL, AL; ${ }^{2}$ Mestrando do Programa de Pós-graduação em Enfermagem, Universidade Federal de Alagoas, UFAL, AL; ${ }^{3}$ Docente do Curso de Graduação em Enfermagem da Universidade Federal de Alagoas, UFAL, AL; ${ }^{4}$ Laboratório de Produtos Naturais e Reabilitação, Universidade Estadual de Ciências da Saúde de Alagoas, UNCISAL, AL, Docente do Mestrado Profissional em Análises de Sistemas Ambientais, Centro Universitário CESMAC, AL; ${ }^{5}$ Docente do Programa de Pósgraduação em Ciências Farmacêuticas, Universidade Federal de Alagoas, UFAL, AL; ${ }^{6}$ Docente do Centro Universitário Tiradentes, UNIT, AL, e do Centro Universitário CESMAC, AL
\end{abstract}

\begin{abstract}
Resumen
Introducción: Cryptococcus neoformans es una levadura encapsulada, que se encuentra en fuentes ambientales, incluyendo excrementos de palomas. Tiene una gran relevancia clínica porque es el agente etiológico de la criptococosis, considerada una causa importante de mortalidad en personas inmunocomprometidas en todo el mundo. Objetivo: determinar la prevalencia y susceptibilidad de C. neoformans aislados del suelo y excremento de palomas en la ciudad de Maceió - Alagoas, Brasil. Metodología: Se recolectaron 150 muestras (50 de excretas secas, 50 de excretas frescas y 50 del suelo) y cultivaron en Agar Dextrosa Sabouraud. Las colonias aisladas sugestivas de C. neoformans se sometieron a identificación y caracterización mediante análisis morfológicos, actividad de la enzima fenol-oxidasa, sensibilidad a la cicloheximida, desarrollo a $37^{\circ} \mathrm{C}$, hidrólisis de urea, asimilación de carbono y nitrógeno y fenotipificación en medio canavanina-glicina-azul de bromotimol. La prueba de susceptibilidad antifúngica se realizó utilizando la técnica de difusión en agar. Resultados: se obtuvieron 36 (24\%) muestras positivas para C. neoformans, de las cuales 33 fueron de excretas secas y 3 del suelo cercano al excremento. El perfil de susceptibilidad fue de 100.0\% para anfotericina B y 87.4\% para ketoconazol, no obstante, hubo un alto porcentaje de resistencia a fluconazol (91.5\%) e itraconazol (80.0\%). Conclusión: estos hallazgos confirman que las excretas de palomas secas son reservorios de C. neoformans en el medio ambiente, caracterizando un problema de salud única. Además, la anfotericina B exhibió una alta actividad in vitro, representando buena alternativa en el tratamiento de la criptococosis. Palabras-clave: Cryptococcus Neoformans. Levadura. Excretas. Palomas. Criptococosis.
\end{abstract}

\begin{abstract}
Resumo
Introdução: Cryptococcus neoformans é um fungo leveduriforme encapsulado, encontrado em fontes ambientais, incluindo excretas de pombos. Apresenta grande relevância clínica por ser agente etiológico da criptococose, considerada importante causa de mortalidade em indivíduos imunocomprometidos em todo o mundo. Objetivo: determinar a prevalência e suscetibilidade de $C$. neoformans isolados do solo e de excretas de pombos na cidade de Maceió, Alagoas. Metodologia: foram coletadas 150 amostras (50 de excretas secas, 50 de excretas frescas e 50 do solo) e cultivadas em Ágar Sabouraud Dextrose. As colônias isoladas sugestivas de $C$. neoformans foram submetidas à identificação e caracterização por meio de análises morfológicas, atividade da enzima fenoloxidase, sensibilidade à cicloheximida, termotolerância à $37^{\circ} \mathrm{C}$, hidrólise da ureia, assimilação de carbono e nitrogênio e quimiotipagem em ágar L-canavanina-glicina-azul de bromotimol. O teste de suscetibilidade antifúngica foi realizado por meio da técnica de difusão em ágar. Resultados: foram obtidas $36(24 \%)$ amostras positivas para $C$. neoformans, das quais $33(91,6 \%)$ foram provenientes de excretas secas e $3(8,3 \%)$ do solo próximo a excrementos. O perfil de suscetibilidade foi de $100,0 \%$ para a anfotericina B e $87,4 \%$ para o cetoconazol, não obstante, verificou-se alto percentual de resistência ao fluconazol (91,8\%) e itraconazol (80\%). Conclusão: estes achados confirmam que excretas de pombos secas são reservatórios de $C$. neoformans no ambiente, caracterizando um problema de saúde única. Além disso, a anfotericina B apresentou elevada atividade, in vitro, representando boa alternativa no tratamento da criptococose.

Palavras-chave: Cryptococcus neoformans. Levedura. Excretas. Pombos. Criptococose.
\end{abstract}

Correspondente/Corresponding: *Rodrigo José Nunes Calumby-Instituto de Ciências Farmacêuticas, Universidade Federal de Alagoas - UFAL, Campus A. C. Simões. End.: Av. Lourival Melo Mota, S/N, Tabuleiro do Martins, Maceió - AL, CEP: 57072-970. Tel: (82) 98842-2479 - E-mail: rjnc_biomed@hotmail.com.

\section{INTRODUCCIÓN}

En los últimos años, el ecosistema urbano se ha vuelto cada vez más favorable a la permanencia de las palomas (Columba livia). Este aumento se debe especialmente la cantidad de alimentos disponibles en entornos públicos, como plazas y parques, lo que conduce a la acumulación 
de excrementos, considerados una fuente importante de infecciones, especialmente la criptococosis en humanos, convirtiéndose en un problema ambiental y de salud única (RIBEIRO et al., 2019).

La criptococosis es una infección fúngica causada por especies del complejo Cryptococcus neoformans/ Cryptococcus gattii (BRILHANTE et al., 2020), de enfoque generalmente pulmonar, pudiendo extenderse, en la mayoría de las veces, al Sistema Nervioso Central (SNC), pero también puede afectar a la piel, mucosas, huesos y vísceras (SIDRIM; ROCHA, 2010; ZAITZ et al., 2010), especialmente en individuos inmunocomprometidos, como pacientes con Síndrome de Inmunodeficiencia Adquirida (AIDS), sarcoidosis y trastornos linfoproliferativos, sometidos a terapias inmunosupresoras (COGLIATI, 2013). También se han registrado informes en pacientes inmunocompetentes, aunque son menos frecuentes (BOLLAM et al., 2020).

La transmisión generalmente ocurre a través de la inhalación de levaduras que alcanzan primeramente el pulmón y, a través de la ruta hematógena, puede extenderse a otros órganos (GENTIL et al., 2016), siendo la meningitis la principal forma clínica de criptococosis diseminada, común en individuos con el Virus de Inmunodeficiencia Humana (HIV), causando la muerte de 180.000 personas en todo el mundo, principalmente en los países en desarrollo (LAKOH et al., 2020). En Brasil, la enfermedad es la segunda causa de muerte entre las micosis sistémicas y tiene una alta tasa de mortalidad (45 a 65\%) (SOARES et al., 2019).

Aunque en el pasado el agente etiológico de la criptococosis se consideraba una especie anamórfica homogénea ( $C$. neoformans), recientemente se han separado dos especies ( $C$. neoformans y $C$. gattii) en base a numerosas diferencias, como la distribución geográfica, nichos ecológicos, epidemiología, patobiología, presentación clínica y características moleculares (COGLIATI, 2013; NWEZE et al., 2015). Sin embargo, la taxonomía y la nomenclatura de estas especies se están revisando, y todavía no hay consenso sobre los cambios de nomenclatura en la comunidad científica (TAVERNA et al., 2020).

La especie C. neoformans es cosmopolita, afecta principalmente pacientes inmunocomprometidos y ha sido ampliamente asociada con excrementos aviares (rico en fuentes de nitrógeno, como urea y creatinina), relacionada con hábitats de palomas en entornos urbanos, aunque también se ha aislada de otras fuentes, como el suelo y las aves cautivas (NWEZE et al., 2015; TAKAHARA et al., 2013). Por otro lado, la especie $C$. gattii está restringida a regiones tropicales y subtropicales, siendo relacionada con huecos de árboles, especialmente eucaliptos (Eucaliptos) y madera en descomposición, involucrando generalmente individuos sin evidencia de inmunosupresión, comportándose como un patógeno primario (ARAÚJO JÚNIOR et al., 2015; BANDALIZADEH et al., 2020; SIDRIM; ROCHA, 2010).
En las excretas de palomas, $C$. neoformans tiene una gran relevancia clínica, ya que son viables en sustratos secos, durante aproximadamente dos años, debido a su capacidad para resistir la desecación. El hongo sobrevive bien cuando no está expuesto directamente a la luz solar y cuando está protegido de las altas temperaturas. La temperatura corporal de las palomas $\left(42^{\circ} \mathrm{C}\right)$, asociada con una mayor actividad de los macrófagos, restringe su crecimiento en estas aves (RIBEIRO et al., 2019).

Datos epidemiológicos indican que la criptococosis ha aumentado en Brasil en las últimas décadas, y que la mayoría de los casos se concentran en las regiones sudeste y sur, con escasez de datos en otras regiones (GENTIL et al., 2016; SOARES et al., 2019). Además, los casos de resistencia a los antifúngicos de amplio espectro son cada vez más frecuentes (BARBOSA JÚNIOR et al., 2015; NAICKER et al., 2020), especialmente a los azoles fluconazol e itraconazol, ampliamente utilizados en el tratamiento de la criptococosis asociada al AIDS, que puede haber promovido una presión selectiva, contribuyendo a la disminución de la susceptibilidad a estos medicamentos (ANDRADE-SILVA et al., 2013).

Por lo tanto, este estudio tuvo como objetivo determinar la prevalencia y susceptibilidad de Cryptococcus neoformans aislados del suelo y excretas de palomas en la ciudad de Maceió - Alagoas, Brasil.

\section{METOdOLOGÍA}

\section{Recolección y procesamiento de muestras}

Para verificar la prevalencia de $C$. neoformans, se recolectaron 50 muestras de excretas secas, constituidas por material deshidratado por efecto del viento y del sol, pero con el mantenimiento de su forma, 50 de excretas frescas, recién eliminadas, y 50 de suelo cercano a los excrementos, utilizándose espátulas de madera desechables y recolectores de plástico estériles con una capacidad de $100 \mathrm{ml}$. Las recolecciones se realizaron en la Plaza dos Palmares ( $\left.9^{\circ} 40^{\prime} 01.0^{\prime \prime} \mathrm{S} 35^{\circ} 44^{\prime} 13.6^{\prime \prime} \mathrm{W}\right)$, Plaza Dom Pedro II ( $\left.9^{\circ} 39^{\prime} 56.6^{\prime \prime} \mathrm{S} 35^{\circ} 44^{\prime} 09.9^{\prime \prime} \mathrm{W}\right)$, Plaza Afrânio Jorge $\left(9^{\circ} 40^{\prime} 01.0^{\prime \prime} \mathrm{S} 35^{\circ} 44^{\prime} 27.5^{\prime \prime} \mathrm{W}\right)$ y en el puerto marítimo $\left(9^{\circ} 40^{\prime} 28.9^{\prime \prime} \mathrm{S} 35^{\circ} 43^{\prime} 15.1^{\prime \prime} \mathrm{W}\right)$, lugares cercanos a calles transitadas y con un importante flujo de personas en la ciudad de Maceió-Alagoas, Brasil. Después de la recolección, las muestras fueron debidamente etiquetadas, empaquetadas y transportadas al Laboratorio de Micología del Centro Universitario Cesmac para continuación de los experimentos.

De cada muestra se pesó aproximadamente $1 \mathrm{~g}$ del material y suspendió en tubos que contenían $9 \mathrm{ml}$ de agua destilada estéril más cloramphenicol $(50 \mathrm{mg} / \mathrm{L})$. Posteriormente, las muestras se agitaron en vórtex durante 3 minutos y se mantuvieron en reposo durante 30 minutos. Transcurrido este tiempo, se retiraron 100 $\mu \mathrm{l}$ del sobrenadante y se cultivaron en placas de Petri que contenían Agar Dextrosa Sabouraud (ASD). Las 
placas se incubaron a temperatura ambiente $\left( \pm 28^{\circ} \mathrm{C}\right)$ y se observaron diariamente durante hasta siete días. Las colonias que parecían lisas, brillantes, mucoides, de color que van desde el blanco a la crema (SIDRIM; ROCHA, 2010; ZAITZ et al., 2010) fueron transferidas a tubos de ensayo que contenían ASD para el mantenimiento de cepas y posterior caracterización.

\section{Caracterización de aislamientos de Cryptococcus}

Los aislamientos se caracterizaron mediante métodos fenotípicos.

El análisis microscópico de las colonias sospechosas se realizó mediante el montaje en un portaobjetos y cubreobjetos utilizando el colorante tinta china y observación bajo un microscopio óptico (aumento de 400X). La identificación de $C$. neoformans se basó en la observación de levaduras regulares, globosas, redondeadas, encapsuladas, con o sin brote y $\sin$ hifas o pseudo-hifas (SIDRIM; ROCHA, 2010; ZAITZ et al., 2010).

La síntesis de pigmentos de melanina a partir de fenol es específica de $C$. neoformans y $C$. gattii. Los medios que contienen semillas de Níger (Guizotia abyssinica) sirven como sustrato para la enzima fenoloxidasa, que cataliza la oxidación de compuestos O-difenólicos como L-dopa, L-dopamina, ácido clorogénico y ácido cafeico, produciendo una colonia marrón oscura o negra en hasta cinco días (SIDRIM; ROCHA, 2010). Además, estas especies de Cryptococcus son capaces de soportar la temperatura corporal (37으) (ARAÚJO JÚNIOR et al., 2015). De esta manera, todos los aislamientos sugestivos de Cryptococcus se cultivaron en placas de Petri que contenían Agar de Semilla de Níger (ASN) para evaluar la producción de melanina en el medio y la consiguiente actividad de fenoxilidasa. Las placas se incubaron a 37 으 y se observaron diariamente durante cinco días. El desarrollo de colonias marrones se consideró un resultado positivo (SIDRIM; ROCHA, 2010).

El crecimiento de Cryptococcus spp. se inhibe en presencia de cicloheximida (ZAITZ et al., 2010). Por lo tanto, los aislamientos se cultivaron en agar Sabouraud suplementado con este antimicrobiano y se incubaron a temperatura ambiente hasta cinco días para evaluar la inhibición de microorganismos en el medio.

La detección de la enzima ureasa, producida por la especie de Cryptococcus, se evaluó utilizando el medio agar base urea. Los aislamientos cultivados en este medio se incubaron a temperatura ambiente hasta cinco días (RIBEIRO et al., 2019). Los resultados obtenidos también se asociaron con pruebas de asimilación y fermentación de fuentes de carbono y nitrógeno (ZAITZ et al., 2010).

Para diferenciar las especies de $C$. neoformans y C. gattii, se utilizó el medio canavanina-glicina-azul de bromotimol (CGB). Las colonias de $C$. gattii usan glicina como fuente de carbono y nitrógeno y son resistentes a la canavanina, presumiendo un color azul cobalto, mientras que $C$. neoformans no muestra un cambio en el color del medio. Los aislamientos se cultivaron en medio CGB y se incubaron a temperatura ambiente hasta cinco días, observando el color del medio diariamente (ARAÚJO JÚNIOR et al., 2015).

\section{Prueba de susceptibilidad antifúngica}

Para evaluar la susceptibilidad a los antimicóticos, se seleccionaron al azar 19 aislamientos de C. neoformans. La susceptibilidad de las cepas se realizó utilizando el método de difusión en agar, de acuerdo con el documento M44-A2 del Clinical \& Laboratory Standards Institute - CLSI (2018) con algunas modificaciones. Se utilizaron cuatro agentes antifúngicos, a saber: anfotericina B (Bristol-M-sanibb), itraconazol (Jassen), ketoconazol (Jassen), disueltos en DMSO (Dimetilsulfóxido) y fluconazol (Pfizer) solubilizado en agua destilada. Se hicieron diluciones en medio de cultivo caldo Sabouraud, a partir de una solución stock de $5000 \mu \mathrm{g} / \mathrm{ml}$ para cada antifúngico, obteniendo cinco concentraciones: $700 \mu \mathrm{g} / \mathrm{ml}, 350 \mu \mathrm{g} / \mathrm{ml}, 150 \mu \mathrm{g} / \mathrm{ml}, 75$ $\mu \mathrm{g} / \mathrm{ml}$ y $37,5 \mu \mathrm{g} / \mathrm{ml}$.

Los inóculos se prepararon en solución salina estéril y la suspensión fúngica se determinó por espectrofotometría equivalente a la de una solución estándar de escala McFarland 0.5. Este procedimiento proporciona una suspensión de levadura que contiene entre 1 y $5 \times 10^{6}$ células $/ \mathrm{ml}$. Para realizar la prueba, se colocaron swabs estériles en las suspensiones fúngicas y, poco después, se sembraron suavemente en todas las direcciones de las placas de Petri que contenían ASD, tratando de cubrir toda la superficie. Las placas sembradas se dejaron reposar durante aproximadamente 5-10 minutos, permitiendo la absorción completa del inóculo por la superficie del medio. Después de este período, se hicieron agujeros de $6 \mathrm{~mm}$ de diámetro con la ayuda de un cilindro para perforar pozos, donde se aplicaron antifúngicos. En cada orificio, se añadieron $20 \mu \mathrm{l}$ de soluciones antifúngicas en cinco concentraciones diferentes. Como control negativo, se usó una solución de DMSO al $2 \%$. Las placas se incubaron a temperatura ambiente durante $24-48$ horas. Todo el experimento se realizó por duplicado. La cepa estándar de Candida albicans ATCC 90028 se usó para controlar el experimento.

La lectura se realizó de acuerdo con las recomendaciones de CLSI (2018), midiendo el halo de inhibición del crecimiento. Tomando como referencia la concentración más baja probada en este estudio (37,5 $\mu \mathrm{g} / \mathrm{ml}$ ) como la Concentración Inhibitoria Mínima (MIC), los antifúngicos se interpretaron de la siguiente manera: Sensible (S) ( $\geq 19 \mathrm{~mm}$ ), Sensible Dependiente de la Dosis (SDS) (halos entre 15 a $18 \mathrm{~mm}$ ) y Resistente (R) ( $\leq 14 \mathrm{~mm}$ ) para cada medicamento.

\section{Análisis estadístico}

El análisis estadístico se realizó con base en el nivel de significación del 95\% ( $p<0.001)$ de los resultados 
obtenidos del aislamiento de $C$. neoformans, utilizando el programa Biostat ${ }^{\circledR}$ versión 5.0.

La concentración inhibitoria mínima del $50 \%\left(\mathrm{CIM}_{50}\right)$ y el $90 \%\left(\mathrm{CIM}_{90}\right)$ se definió como la cantidad necesaria para inhibir, respectivamente, $50 \%$ y $90 \%$ de los aislamientos analizados. El porcentaje de actividad y concentraciones de antimicóticos se relacionó utilizando el programa "Microsoft Excel 2016", obteniendo, para cada antifúngico, la ecuación de línea. La resolución de esa ecuación (reemplazando el valor de $\mathrm{Y}$ con 50 o 90) resultó en el valor de $\mathrm{CIM}_{50}$ y $\mathrm{CIM}_{90}$.

\section{RESULTADOS}

\section{Caracterización morfológica y bioquímica de los aislamientos}

Todos los aislados sugestivos de $C$. neoformans en ASD se presentaron como colonias mucosas, viscosas, brillantes, lisas y blanquecinas, capaces de producir melanina en ASN, que corresponde a un importante factor de virulencia. En el análisis microscópico, utilizando tinta China, se verificaron levaduras ovales o globosas, encapsuladas, con o sin brotación, sin hifas o pseudo-hifas. Todas fueron tolerantes a 37 으, produciendo ureasa, inhibida por la cicloheximida. El perfil de asimilación de carbono y nitrógeno demostró que todas las cepas asimilaron dextrosa, sacarosa, maltosa, trehalosa, dulcitol, inositol, D-xilosa y peptona y no asimilaron lactosa, melibiosis y nitrato, confirmando las características básicas de identificación de este hongo. El cultivo en el medio CGB fue negativo para todos los aislamientos, lo que demuestra que pertenecían a la especie $C$. neoformans.
Aislamiento de C. neoformans de muestras de suelo y excretas de palomas

Entre las 150 muestras de suelo y excretas de palomas evaluadas, $36(24.0 \%)$ cultivos fueron positivos para $C$. neoformans, 33 de los cuales provenían de excretas secas ( $p<0.001$ ) y tres del suelo. No fue posible aislar el hongo en excretas frescas, como se muestra en la Tabla 1.

Tabla 1 - Distribución de muestras positivas y negativas de C. neoformans aislados del suelo y excretas de palomas en la ciudad de Maceió - AL, Brasil.

\begin{tabular}{lccccc}
\hline & \multicolumn{3}{c}{ Aislamiento de C. neoformans } \\
\cline { 2 - 3 } Muestras & \multicolumn{2}{c}{ Positivo } & & \multicolumn{2}{c}{ Negativo } \\
\cline { 2 - 3 } \cline { 5 - 6 } & $n$ & $\%$ & & $n$ & $\%$ \\
\hline Suelo $(n=50)$ & 3 & 6.0 & & 47 & 94.0 \\
Excretas frescas $(n=50)$ & - & - & & 50 & 100.0 \\
Excretas secas $(n=50)$ & $33^{*}$ & 66.0 & & 17 & 34.0 \\
\hline Total $(n=150)$ & $\mathbf{3 6}$ & $\mathbf{2 4 . 0}$ & & 114 & 76.0 \\
\hline
\end{tabular}

Leyenda: $n=$ frecuencia absoluta; \% = frecuencia relativa.

${ }^{*} p<0.001$.

Source: El autor.

De las 33 muestras de excremento seco positivas para C. neoformans, la mayor prevalencia se observó en el Puerto de Maceió (12), seguido de la Plaza dos Palmares (10), Plaza Dom Pedro II (8) y Plaza Afrânio Jorge (3). Con respecto a las muestras recolectadas del suelo, los 3 cultivos positivos provinieron del Puerto de Maceió, como se muestra en la tabla 2.

Tabla 2 - Frecuencia de aislamiento de C. neoformans del suelo y excremento seco de palomas según el sitio de recolección en la ciudad de Maceió - AL, Brasil.

\begin{tabular}{|c|c|c|c|c|c|c|}
\hline \multirow{2}{*}{ Localizaciones } & \multirow{2}{*}{$\begin{array}{l}\text { Número de excrementos } \\
\text { secos recolectados }\end{array}$} & \multicolumn{2}{|c|}{ Aislamiento de excretas } & \multirow{2}{*}{ Número de muestras del suelo } & \multicolumn{2}{|c|}{ Aislamiento del suelo } \\
\hline & & Positivo & Negativo & & Positivo & Negativo \\
\hline Plaza dos Palmares & 12 & 10 & 2 & 12 & - & 12 \\
\hline Plaza Dom Pedro II & 12 & 8 & 4 & 12 & - & 12 \\
\hline Plaza Afrânio Jorge & 12 & 3 & 9 & 12 & - & 12 \\
\hline Puerto de Maceió & 14 & 12 & 2 & 14 & 3 & 11 \\
\hline TOTAL & 50 (100.0\%) & $33(66.0 \%)$ & $17(34.0 \%)$ & 50 (100.0\%) & $3(6.0 \%)$ & 47 (94.0\%) \\
\hline
\end{tabular}

Source: El autor.

\section{Perfil de susceptibilidad a los medicamentos} antifúngicos

Las muestras de $C$. neoformans identificadas en este estudio mostraron un alto porcentaje de resistencia al itraconazol (80\%) y al fluconazol (91.5\%), con altos valores de $\mathrm{CIM}_{50}$ y $\mathrm{CIM}_{90}$. Para fluconazol, el cálculo de CIM 50\% y $90 \%$ se volvió inviable, ya que la mayor concentración de este fármaco no fue capaz de inhibir el $50 \%$ de las especies. En contraste, el perfil de sensibilidad para anfotericina B (100.0\%) y ketoconazol (87.4\%) fueron altos. Ambas drogas ofrecieron inhibición de las cepas a la concentración más baja probada, haciendo que la definición de MIC 50 sea inviable, como se muestra en la tabla 3. 
Tabla 3 - Perfil de susceptibilidad a los medicamentos antifúngicos de C. neoformans $(n=19)$ aislados del suelo y excretas de palomas en la ciudad de Maceió - AL, Brasil.

\begin{tabular}{|c|c|c|c|c|c|c|}
\hline \multirow{2}{*}{ Antifúngico } & \multirow{2}{*}{ Variación ( $\mu \mathrm{g} / \mathrm{ml})$} & \multicolumn{2}{|c|}{$\mathrm{CIM}(\mu \mathrm{g} / \mathrm{ml})$} & \multicolumn{3}{|c|}{ Perfil de susceptibilidad } \\
\hline & & $\mathrm{CIM}_{50}$ & $\mathrm{CIM}_{90}$ & S (\%) & SDD (\%) & R (\%) \\
\hline Anfotericina B & 37,5 a 350 & $<37,5$ & 325 & 100,0 & - & - \\
\hline Ketoconazol & 37,5 a 700 & $\leq 37,5$ & 336 & 87,4 & 10,5 & 2,1 \\
\hline Itraconazol & $350 a \geq 700$ & 478 & 708 & 19,0 & 1,0 & 80,0 \\
\hline Fluconazol & $\geq 700$ & $\geq 700$ & $>700$ & 3,2 & 5,3 & 91,5 \\
\hline
\end{tabular}

Leyendas: $\mathrm{CIM}_{50}$ y $\mathrm{CIM}_{90}=$ concentración necesaria para inhibir, respectivamente, $50 \%$ y $90 \%$ de los aislamientos.); S (sensible); SDD (sensible dependiente de la dosis); $R$ (resistente).

Source: El autor.

\section{DISCUSIÓN}

Este estudio evidenció el aislamiento de C. neoformans en excrementos secos de palomas, confirmando que este sustrato es una fuente importante del hongo en el ambiente urbano, concordante con los resultados de otras investigaciones realizadas en Brasil y en otros países. En este seguimiento, Ribeiro et al. (2019) verificaron la presencia de $C$. neoformans en las excretas de palomas urbanas en el municipio de Redenção AM, Brasil, y obtuvieron $16 \%$ de aislamiento en estos sustratos en 10 vecindarios estudiados. En otro estudio, Krangvichain et al. (2016), determinaron la presencia de este patógeno en excrementos de palomas en la ciudad de Bangkok, Tailandia, y encontraron un porcentaje de positividad de $17.5 \%$ en excremento seco. Resultados inferiores a los encontrados en este estudio, en el cual se verificó un porcentaje del $66.0 \%$ para esta muestra. Otros estudios también han verificado la presencia de $C$. neoformans en excretas secas de palomas, con porcentajes de $6.6 \%$ (TAKAHARA et al., 2013), 10.0\% (TANGWATTANACHULEEPORN et al., 2013), 22.0\% (NWEZE et al., 2015), 26.9\% (FARIA et al., 2010) y 66.6\% (TEODORO et al., 2013).

Por otro lado, no fue posible aislar a C. neoformans en excretas frescas de palomas, lo que coincide con los hallazgos de otros autores (FARIA et al., 2010; KRANGVICHAIN et al., 2015) y que puede justificarse por la proliferación bacteriana intensa en estos excrementos, promoviendo un cambio en el pH, haciéndolo alcalino, inhibiendo el crecimiento de la levadura (FARIA et al., 2010). De acuerdo con Ribeiro et al. (2019), el excremento seco ofrece un sustrato orgánico más propicio para el desarrollo del hongo, rico en nitrógeno, con una menor concentración de bacterias, lo que reduce la competencia por el crecimiento. El desarrollo de $C$. neoformans en estos sustratos también favorece la formación de microfocos en los centros urbanos, haciendo que la exposición de los humanos a este patógeno sea una ocurrencia común, especialmente en el polvo doméstico (REOLON et al., 2004). La presencia de $C$. neoformans en las excretas de estas aves también puede variar según la dieta, las condiciones de mantenimiento del animal, el comportamiento de cada especie y los aspectos climáticos de cada región (SIDRIM; ROCHA, 2010).

La frecuencia de aislamiento de $C$. neoformans del suelo fue de $6.0 \%$ en las 50 muestras analizadas, resultado superior que el encontrado por Yamamura et al. (2013), que verificaron la presencia de $C$. neoformans en el 0,54\% de las muestras de suelo con excrementos de palomas, e inferior que lo evidenciado en el estudio de Machado et al. (1993), que obtuvieron $27,11 \%$ de muestras positivas para este mismo sustrato. C. neoformans fue aislado del suelo por primera vez por Emmons en 1951, y posteriormente se comprobó un vínculo estrecho entre el aislamiento del suelo y los nidos de palomas, lo que demuestra que la presencia del hongo se debe principalmente a la concentración de excrementos en el local (SIDRIM; ROCHA, 2010).

El alto índice de muestras positivas en el Puerto de Maceió indica este lugar como un importante reservorio de palomas y una alta proliferación de $C$. neoformans en el medio ambiente. Durante las recolecciones, se observó una alta concentración de estas aves y excrementos en este ambiente, con varios nidos presentes en los cobertizos del establecimiento, lo que evidencia la necesidad de medidas de control ambiental. La Plaza Dom Pedro II es otro lugar favorable para la proliferación de palomas, ya que es un ambiente que abarca la mayor cantidad de fuentes de alimento, promoviendo la supervivencia y multiplicación de estas aves y, en consecuencia, la exposición de los propágulos fúngicos a personas que transitan en el lugar. Además, la presencia de muestras positivas en la plaza Afrânio Jorge merece atención por su cercanía al entorno hospitalario.

Según Sidrim y Rocha (2010), en las excretas de palomas contaminadas con C. neoformans en altas concentraciones, sus propágulos fúngicos pueden dispersarse en el aire y, posteriormente, inhalarse. La inhalación en grandes cantidades puede desencadenar un proceso infeccioso en inmunocomprometidos y, más raramente, en inmunocompetentes (BOLLAM et al., 
2020; COGLIATI, 2013), pudiendo propagarse y llegar al Sistema Nervioso Central (SNC), donde este hongo tiene una alta afinidad debido a la presencia, en alta concentración, de nutrientes asimilables como tiamina, ácido glutámico, glutamina, dopamina, carbohidratos y minerales, presentes en el líquido cefalorraquídeo (LCR) y la respuesta inflamatoria débil o ausente del tejido cerebral (REOLON et al., 2004). Todavía en este contexto, Queiroz et al. (2008) describen que la cantidad de anticuerpos circulantes después de la exposición a sitios contaminados con propágulos de este hongo es grande, sin embargo, la mayoría de las personas no desarrollan la enfermedad, pero pueden mantener este patógeno en el cuerpo durante mucho tiempo.

Debido a la importancia de las excretas de palomas como reservorio y fuente de infección para C. neoformans, son necesarias medidas de control ambiental. En este sentido, se sugiere la aplicación de acciones dirigidas a minimizar la exposición a este patógeno, mediante el control de palomas en centros urbanos, con un flujo intenso de personas, y en domicilio de pacientes inmunocomprometidos, mediante el monitoreo de las condiciones de higiene y limpieza de excreta, aireación adecuada, iluminación y ventilación, así como monitoreo de sitios de riesgo (REOLON et al., 2004). La simple limpieza del ambiente, eliminando el resto de excrementos puede ser eficiente. Además, se debe evitar el barrido de excretas para impedir la propagación de esporas de hongos en el aire, así como evitar ofrecer comida a estas aves (TAKAHARA et al., 2013).

La resistencia de $C$. neoformans a los antifúngicos azoles fluconazol e itraconazol se ha informado en otros estudios. Drummond et al. (2007) evaluaron la susceptibilidad de varias cepas de $C$. neoformans a esta clase de antifúngicos y evidenciaron que los aislamientos ambientales aumentaron la resistencia a estos medicamentos, suponiendo que la menor susceptibilidad se debe a la posible contaminación ambiental de estos compuestos. En otro estudio, Barbosa Júnior et al. (2015) examinaron la susceptibilidad de los aislamientos clínicos de $C$. neoformans a los agentes antifúngicos para uso hospitalario y observaron una menor sensibilidad al fluconazol e itraconazol, siendo necesaria una concentración de $10 \mathrm{mg} / \mathrm{ml}$ (itraconazol) y $12 \mathrm{mg} / \mathrm{ml}$ (fluconazol) para promover la inhibición de la mayoría de aislamientos evaluados.

Naicker et al. (2020) estudiaron la disminución de la susceptibilidad de los aislados clínicos de $C$. neoformans al fluconazol durante una década en Sudáfrica y comprobaron que los valores de MIC 50 y 90 eran dos veces más altos en 2017 en comparación con los años 2007 y 2008 , lo que sugiere que pueden ser necesarias dosis más altas de fluconazol para mantener la efectividad de los protocolos de tratamiento para la meningitis criptocóccica. Menor susceptibilidad al fluconazol también se observó en los estudios de Alves et al. (2016) y Córdoba et al. (2016).
En lo que concierne al itraconazol, este antimicótico se ha utilizado durante mucho tiempo como una alternativa terapéutica al fluconazol en el tratamiento de la criptococosis, especialmente en formas que no alcanzan al SNC (SIDRIM; ROCHA, 2010). Sin embargo, algunos estudios han encontrado cepas resistentes a este medicamento con valores altos de CIM (BARBOSA JUNIOR et al., 2015; FAVALESSA et al., 2009), lo que sugiere que su reemplazo con fluconazol debe evaluarse cuidadosamente. Aunque la actividad in vitro de fluconazol es baja en algunos estudios, se cree que in vivo este antifúngico puede alcanzar niveles plasmáticos más altos que el itraconazol (SILVA et al., 2008).

Diferente de estos resultados, Teodoro et al. (2013) determinaron la susceptibilidad de $C$. neoformans aislado de excretas de palomas en la ciudad de Araraquara - SP y no identificaron aislamientos resistentes, concordante con otros estudios, como los de Souza et al. (2010), quienes evaluaron la susceptibilidad de los aislamientos ambientales de $C$. neoformans en la ciudad de Goiânia - GO y la de Tangwattanachuleeporn et al. (2013) quienes investigaron la susceptibilidad antifúngica de $C$. neoformans aislados de excrementos de palomas en la provincia de Chon Buri, este de Tailandia.

En nuestro estudio, todas las cepas evaluadas demostraron susceptibilidad a la anfotericina B, coincidiendo con otros estudios (BANDALIZADEH et al., 2020; FAVALESSA et al., 2009). Este medicamento se ha usado con frecuencia en el tratamiento de la criptococosis diseminada en asociación con otros antifúngicos (SIDRIM; ROCHA, 2010) y la detección de cepas ambientales de $C$. neoformans resistentes a anfotericina $B$ es poco común, lo que sugiere que su sensibilidad puede ser el resultado de la falta de exposición a este medicamento, administrado exclusivamente en hospitales (ANDRADESILVA et al., 2013), sin embargo, este patrón no siempre se observa en cepas clínicas (SMITH et al., 2015). En este sentido, Andrade-Silva et al. (2013) evaluaron el perfil de sensibilidad de los aislamientos clínicos y ambientales de C. neoformans en Uberaba, Minas Gerais, y observaron que un total del $10.5 \%$ de los aislamientos clínicos de esta especie era resistente a la anfotericina B. Resultados similares también fueron observados por Barbosa Junior et al. (2015).

El desarrollo de resistencia a la anfotericina $B$ en cepas hospitalarias puede estar relacionado con la recuperación de aislamientos de pacientes con AIDS, que a menudo están expuestos a antifúngicos azoles, especialmente fluconazol, para prevenir y tratar infecciones fúngicas (ANDRADE-SILVA et al., 2013). La exposición previa al fluconazol puede haber inducido una menor susceptibilidad debido a una reducción en la cantidad de ergosterol en la membrana plasmática o un cambio en el esterol diana, lo que lleva a una disminución en la ligación de anfotericina B (SPADARI et al., 2020).

Con respecto al ketoconazol, solo el $2.1 \%$ de los aislamientos presentaron resistencia en este estudio, 
similar a lo reportado por otros autores que también demostraron una alta susceptibilidad de $C$. neoformans a este medicamento (ALVES et al., 2016; KHAN et al., 2007). Sin embargo, el ketoconazol no se usa con frecuencia en la práctica clínica para el tratamiento de infecciones sistémicas como la criptococosis (SIDRIM; ROCHA, 2010). De acuerdo con Zaitz et al. (2010), el uso de este antifúngico para el tratamiento de la meningitis criptocóccica no genera resultados satisfactorios, ya que este medicamento no presenta una buena penetración en el SNC y no alcanza concentraciones terapéuticas efectivas sin la aparición de efectos adversos.

\section{CONCLUSIÓN}

C. neoformans se aisló de excretas secas de palomas, lo que demuestra que estos excrementos funcionan como un ambiente rico en nutrientes para el crecimiento y la propagación del hongo en el medio ambiente, lo que puede representar una fuente importante de infección, especialmente para individuos inmunocomprometidos. Aunque con resultados mínimos, también se obtuvieron muestras positivas en el suelo cerca del excremento. Estos hallazgos demuestran la necesidad de medidas para controlar estas aves en lugares de circulación pública, como plazas y parques, así como la aplicación de prácticas de higienización y limpieza de las excretas.

Con respecto a la susceptibilidad antifúngica, se observó una baja sensibilidad de $C$. neoformans a los antifúngicos azoles fluconazol e itraconazol, con altos valores de CIM, lo que demuestra una resistencia significativa en las concentraciones probadas $y$, en consecuencia, una disminución en las opciones de tratamiento. En contraste, el fármaco anfotericina $B$ mostró una alta actividad in vitro, lo que demuestra la eficacia de este fármaco en la inhibición de aislamientos de C. neoformans y en el tratamiento de la criptococosis.

\section{REFERENCIAS}

ALVES, G. S. et al. Molecular typing of environmental Cryptococcus neoformans/C. gattii species complex isolates from Manaus, Amazonas, Brazil. Mycoses, Berlin, v. 59, n. 8, p. 509-515, 2016.

ANDRADE-SILVA, L. et al. Susceptibility profile of clinical and environmental isolates of Cryptococcus neoformans and Cryptococcus gattii in Uberaba, Minas Gerais, Brazil. Med. Mycol., Oxford, v. 51, n. 6, p. 635-640, 2013.

ARAÚJO JÚNIOR, E. C. et al. Cryptococcus: isolamento ambiental e caracterização bioquímica. Arq. Bras. Med. Vet. Zootec., Belo horizonte, v. 67, n. 4, p. 1003-1008, 2015.

BANDALIZADEH, Z. et al. Molecular epidemiology and antifungal susceptibility profiles of clinical Cryptococcus neoformans/Cryptococcus gattii species complex. J. Med. Microbiol., Mumbai, v. 69, n. 1, p. 7281, 2020.

BARBOSA JUNIOR, A. M. et al. Estudo comparativo da susceptibilidade de isolados clínicos de Cryptococcus neoformans (Sanfelice, 1895) frente a alguns antifúngicos de uso hospitalar e extratos vegetais obtidos de plantas medicinais da região semiárida sergipana. Rev. Bras. Plantas Med., Botucatu, v. 17, n. 1, p. 120-132, 2015.
BOLLAM, R. et al. Disseminated cryptococcosis in an immunocompetent patient. Respir. Med. Case Rep., [s.I], v. 30, p. 1-3, 2020.

BRILHANTE, R. S. N. et al. Cryptococcus neoformans/Cryptococcus gattii species complex melanized by epinephrine: increased yeast survival after amphotericin B exposure. Microb. Pathog., London, v. 143, n. 104123, 2020.

CLINICAL AND LABORATORY STANDARDS INSTITUTE (CLSI). Method for antifungal disk diffusion susceptibility testing of yeasts: approved guideline M44-A2. CLSI, Wayne, PA, USA, 2018.

COGLIATI, M. Global molecular epidemiology of Cryptococcus neoformans and Cryptococcus gattii: an atlas of the molecular types. Scientifica (Cairo), v. 2013, p. 1-23, 2013.

CÓRDOBA, S. et al. Susceptibility profile and epidemiological cut-off values of Cryptococcus neoformans species complex from Argentina. Mycoses, Berlin, v. 59, n. 6, p. 351-356, 2016.

DRUMMOND, E. D. et al. Comportamento de amostras ambientais e clínicas de Cryptococcus neoformans frente a fungicidas de uso agronômico e ao fluconazol. Rev. Soc. Bras. Med. Trop., Brasilia, v. 40, n. 2, p. 209-211, 2007.

FARIA, R. O. et al. Ocorrência de Cryptococcus neoformans em excretas de pombos na cidade de Pelotas, Estado do Rio Grande do Sul. Rev. Soc. Bras. Med. Trop., Brasilia, v. 43, n. 2, p. 198-200, 2010.

FAVALESSA, O. C. et al. Primeira descrição da caracterização fenotípica e susceptibilidade in vitro a drogas de leveduras do gênero Cryptococcus spp isoladas de pacientes HIV positivos e negativos, Estado de Mato Grosso. Rev. Soc. Bras. Med. Trop., Brasilia, v. 42, n. 6, p. 661-665, 2009.

GENTIL, F. A. et al. Criptococose: relato de caso. Acta Biomed. Bras., [s.I], v. 7, n. 2, p. 102-109, 2016.

KHAN, Z. U. et al. Antifungal susceptibility of Cryptococcus neoformans and Cryptococcus gattii isolates from decayed wood of trunk hollows of Ficus religiosa and Syzygium cumini trees in north-western India. J. Antimicrob. Chemother., London, v. 60, n. 2, p. 312-316, 2007.

KRANGVICHAIN, P. et al. Occurrence and susceptibilities to disinfectants of Cryptococcus neoformans in fecal droppings from pigeons in Bangkok, Thailand. J. Vet. Med. Sci., Tokyo, v. 78, n. 3, p. 391-396, 2016.

LAKOH, S. et al. Prevalence and mortality of cryptococcal disease in adults with advanced HIV in an urban tertiary hospital in Sierra Leone: a prospective study. BMC Infect. Dis., London, v. 20, n. 141, p. 1-12, 2020.

MACHADO, C. C. et al. Cryptococcus neoformans var. neoformans isolado do solo. Rev. Inst. Med. Trop. Sao Paulo, São Paulo, v. 35, n. 1, p. 77-79, 1993.

NAICKER, S. D. et al. Decreasing fluconazole susceptibility of clinical South African Cryptococcus neoformans isolates over a decade. PLoS Negl. Trop. Dis., San Francisco, v. 14, n. 3, p. 1-11, 2020.

NWEZE, E. I. et al. Isolation of Cryptococcus neoformans from environmental samples collected in Southeastern Nigeria. Rev. Inst. Med. Trop. Sao Paulo, São Paulo, v. 57, n. 4, p. 295-298, 2015.

QUEIROZ, J. P. A. F. et al. Criptococose - uma revisão bibliográfica. Acta Vet. Bras., [s.I], v. 2, n. 2, p. 32-38, 2008.

REOLON, A. et al. Prevalência de Cryptococcus neoformans nos pombos urbanos da cidade de Porto Alegre, Rio Grande do Sul. J. Bras. Patol. Med. Lab., Rio de Janeiro, v. 40, n. 5, p. 293-298, 2004.

RIBEIRO, E. A. et al. Occurrence of Cryptococcus neoformans in the excreta of urban pigeons in the municipality of Redenção in Amazônia, Brazil. Acta Biomed. Bras.,[s.I], v. 10, n. 1, p. 27-34, 2019. 
SIDRIM, J. J. C.; ROCHA, M. F. G. Micologia Médica à luz de autores contemporâneos. Rio de Janeiro: 2aㅡ. Ed. Guanabara Koogan, 2010.

SILVA, P. R. et al. Suscetibilidade a antifúngicos de variedades de Cryptococcus neoformans isoladas de pacientes em hospital universitário. Rev. Soc. Bras. Med. Trop., Brasília, v. 41, n. 2, p. 158162, 2008.

SMITH, K. D. et al. Increased antifungal drug resistance in clinical isolates of Cryptococcus neoformans in Uganda. Antimicrob. Agents Chemother.,Washington, v. 59, n. 12, p. 7197-7204, 2015.

SOARES, E. A. et al. Mortality by cryptococcosis in Brazil from 2000 to 2012: A descriptive epidemiological study. PLoS Negl. Trop. Dis., San Francisco, v. 13, n. 7, p. 1-17, 2019.

SOUZA, L. K. et al. Molecular typing and antifungal susceptibility of clinical and environmental Cryptococcus neoformans species complex isolates in Goiania, Brazil. Mycoses, London, v. 53, n. 1, p. 62-67, 2010.

SPADARI, C. C. et al. New approaches for cryptococcosis treatment. Microorganisms, [s.I], v. 8, n. 4, p. 1-15, 2020.

TAKAHARA, D. T. et al. First report on Cryptococcus neoformans in pigeon excreta from public and residential locations in the metropolitan area of
Cuiabá, State of Mato Grosso, Brazil. Rev. Inst. Med. Trop. Sao Paulo, São Paulo, v. 55, n. 6, p. 371-376, 2013.

TANGWATTANACHULEEPORN, $M$. et al. Prevalence and antifungal susceptibility of Cryptococcus neoformans isolated from pigeon excreta in Chon Buri Province, Eastern Thailand. Med. Mycol. J., [s.I], v. 54, n. 3, p. 303-307, 2013.

TAVERNA, C. G. et al. Frequency and geographical distribution of genotypes and mating types of Cryptococcus neoformans and Cryptococcus gattii species complexes in Argentina. Rev. Argent. Microbiol., Buenos Aires, v. 52, n. 3, p. 183-188, 2020.

TEODORO, V. L. I. et al. Environmental isolation, biochemical identification, and antifungal drug susceptibility of Cryptococcus species. Rev. Soc. Bras. Med. Trop., Brasília, v. 46, n. 6, p. 759-764, 2013.

YAMAMURA, M. et al. Estudo dos nichos ecológicos de leveduras patogênicas das espécies Cryptococcus neoformans e Cryptococcus gattii na cidade de Londrina, PR. Semina: Ciênc. Agrár., Londrina, v. 34, n. 2, p. 793-804, 2013.

ZAITZ, C. et al. Compêndio de micologia médica. 2. ed. São Paulo: Médica e Científica, 2010.

Submetido em: $27 / 05 / 2020$

Aceito em: 12/03/2021 